\title{
Experiencias del profesorado acerca del aprendizaje autónomo en estudiantes de modalidad a distancia y el uso de recursos digitales
}

\author{
Teachers' Experiences About the Autonomous Learning in Distance Learning \\ Students and the Use of Digital Resources
}

\section{Experiências de professores sobre aprendizagem autônoma em estudantes a distância e uso de recursos digitais \\ José de Jesús Peinado Camacho \\ Instituto Politécnico Nacional, México \\ jpeinadoc@ipn.mx \\ https://orcid.org/0000-0002-2262-4565}

\section{Resumen}

El objetivo de esta investigación fue documentar experiencias del aprendizaje autónomo desde la perspectiva de los profesores de educación a distancia. La metodología que se utilizó fue cualitativa. La investigación fue no experimental con diseño transversal y exploratorio. Se seleccionó el estudio de caso como técnica para obtener la información. La muestra fue no probabilística y heterogénea. Los resultados de la investigación proponen que los profesores fomentan el aprendizaje autónomo al permitir que los alumnos intervengan en la construcción de procedimientos y recursos para alcanzar el propósito y las habilidades planteadas en una actividad específica, de esta manera el estudiante toma conciencia sobre sus decisiones para dirigir y regular su propio aprendizaje. Promover el pensamiento crítico y reflexivo, permitir la autodirección y la autorregulación, aumentar el aprendizaje significativo y emplear habilidades metacognitivas son algunas de las ventajas que los docentes entrevistados perciben al momento de desarrollar el aprendizaje autónomo en los alumnos. 


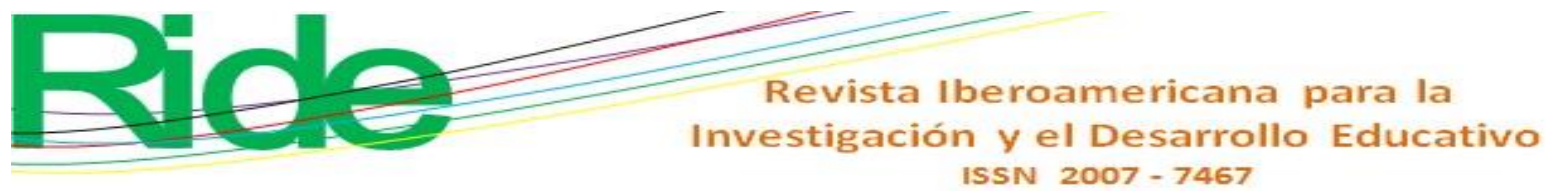

Palabras clave: alumnos, aprendizaje autónomo, educación a distancia, profesores, recursos digitales.

\begin{abstract}
The objective of this research was to document experiences of autonomous learning from the perspective of distance education teachers. The methodology that was used was qualitative. The research was non-experimental with cross-sectional and exploratory design. The case study was selected as a technique to obtain the information. The sample was non-probabilistic and heterogeneous. The results of the research propose that teachers encourage autonomous learning by allowing students to intervene in the construction of procedures and resources to achieve the purpose and skills raised in a specific activity, in this way the student becomes aware of their decisions to direct and regulate their own learning. Promoting critical and reflective thinking, allowing self-direction and self-regulation, increasing meaningful learning and using metacognitive skills, are some of the advantages that the interviewed professors perceive when developing autonomous learning in students.
\end{abstract}

Keywords: students, autonomous learning, distance education, teachers, digital resources.

\title{
Resumo
}

O objetivo desta pesquisa foi documentar experiências de aprendizagem autônoma na perspectiva de professores de educação a distância. A metodologia utilizada foi qualitativa. A pesquisa não foi experimental, com delineamento transversal e exploratório. O estudo de caso foi selecionado como a técnica para obter as informações. A amostra foi não probabilística e heterogênea. Os resultados da pesquisa propõem que os professores promovam a aprendizagem autônoma, permitindo que os alunos intervenham na construção de procedimentos e recursos para atingir o objetivo e as habilidades estabelecidas em uma atividade específica, para que o aluno tome consciência de suas decisões em dirigir e regular sua própria aprendizagem. Promover o pensamento crítico e reflexivo, permitir a auto-direção e a auto-regulação, aumentar a aprendizagem significativa e empregar habilidades metacognitivas são algumas das vantagens que os professores entrevistados percebem ao desenvolver a aprendizagem autônoma nos alunos.

Palavras-chave: estudantes, aprendizagem autônoma, educação a distância, professores, recursos digitais. 


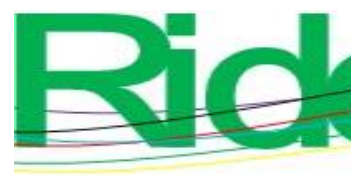

Fecha Recepción: Diciembre 2019

\section{Fecha Recepción: Diciembre 2019}

Revista Iberoamericana para la

Investigación y el Desarrollo Educativo

ISSN $2007-7467$

Fecha Aceptación: Abril 2020

\section{Introducción}

Clarence (2017) determina que "los profesores aceptan que las actividades académicas que son desafiantes pueden despertar los objetivos de rendimiento de los alumnos" (p. 55). Hromalik y Koszalka (2018) coinciden en que "hay mayor compromiso en el aprendizaje autorregulado" (p. 528). Según Chen, Bennett y Maton (2008), "los alumnos se adaptan mejor al aprendizaje en un entorno flexible" (p. 307).

La naturaleza progresiva, continua y orientada a los procesos de diseño de los contenidos es muy importante (Esteban y Zapata, 2016, p. 1; Sun, 2016, p. 349). En este sentido, Park y Yun (2017) mencionan que "los profesores pueden emplear estrategias que mejoren la autoeficacia del aprendizaje en línea" (p. 302). Alqurashi (2019) señala que "debe existir la interacción del alumno con el contenido y con el profesor" (p. 133). Grover y Troja (2014), por su parte, establecen que “al mismo tiempo se puede determinar la satisfacción del alumno y el aprendizaje percibido" (p. 90).

Algunos especialistas como Hromalik y Koszalka (2018) especifican que "monitorear el desempeño, los métodos de aprendizaje, el uso del tiempo y la motivación, son de gran utilidad en el aprendizaje de los estudiantes" (p. 528). Pero se debe considerar que los estudiantes difieren en el uso de estrategias de aprendizaje, estrategias regulatorias, motivación y actitudes hacia una asignatura específica (Clarence, 2008, p. 439; Esteban y Zapata, 2016, p. 1). Por tal motivo, se debe examinar el papel de la orientación a los objetivos y la autoeficacia académica en el rendimiento de los estudiantes influenciado por la regulación del esfuerzo, la regulación metacognitiva y la regulación de la interacción, y desarrollar así el pensamiento crítico y los conocimientos que los propios alumnos muestran a fin de orientar el apoyo donde más se necesita (Cho y Shen, 2013, p. 290; Onrubia, 2016, p. 3; Park y Yun, 2017, p. 302).

Para Clarence (2017) "los alumnos perciben mejor los objetivos enfocados al desempeño para tratar con tareas académicas que requieren que demuestren su competencia” (p. 55). Mientras que Niño, Castellanos y Viloria (2019) precisan que "los estudiantes deben tener la posibilidad de plantear metas a corto y mediano plazo para el desarrollo eficaz de una tarea" (p. 18). De esta manera se logran mejores resultados que con objetivos de dominio en tópicos específicos (Clarence, 2008, p. 439). Adicionalmente, completan sus tareas, demuestran dominio de 


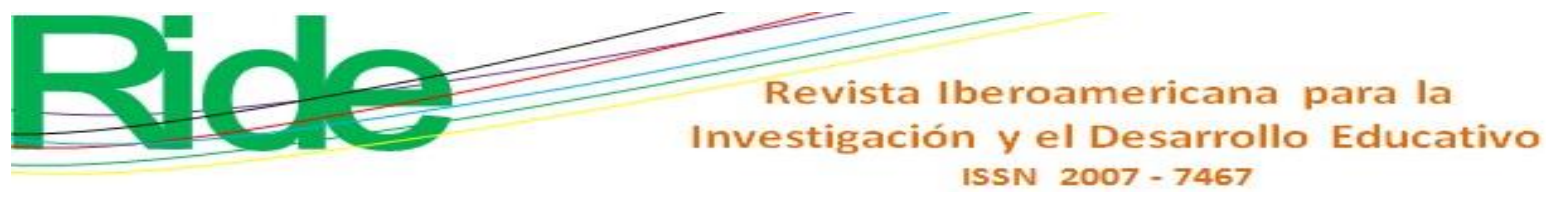

habilidades y son más positivos al momento de tener opciones (Hanewicz, Platt y Arendt, 2017, p. 273).

El acompañamiento en el proceso de formación del estudiante es vital para la construcción de conocimiento (Henning y Escofet, 2015, p. 5). La motivación, la responsabilidad y la retroalimentación juegan un papel crucial en el éxito del autoaprendizaje (MacDougall, 2008, p. 228). Además, para lograr dicho éxito, el alumno debe disponer de habilidades metacognitivas (Onrubia, 2016, p. 3), así como de autoeficacia académica e independiente (Cho y Shen, 2013, p. 291; García, 2012, p. 20). Sin duda se trata de un proceso de preparación para la vida laboral, donde, llegado el momento, el alumno podrá beneficiarse del autoconocimiento en esta etapa alcanzado.

Asimismo, los estudiantes también utilizan el espacio de trabajo colaborativo, al igual que las herramientas y medios digitales para realizar actividades independientes y colaborativas (Amador y Velarde, 2019, p. 8; Henning y Escofet, 2015, p. 8). Esto incluye conocerse entre sí, compartir expectativas personales y grupales, respetar las diferencias individuales, negociar el significado con los demás y autorregularse (Mendieta, Estrada y Pérez, 2019, p. 5; Niño et al., 2019, p. 18), lo cual contribuye al desarrollo de los alumnos en lo individual y a los logros académicos en el curso (Cardoso y Cerecedo, 2019, p. 16; Cho y Shen, 2013, p. 290).

Por otra parte, son variados y extensos los esfuerzos por incorporar nuevas tendencias en la dinámica del aprendizaje virtual con la intención de adaptar propuestas novedosas de medios digitales (Domínguez, Organista y López, 2018; Gros, 2016; Torres, Jara y Valdivieso, 2012); tal es el caso de las plataformas de mensajería, mejor conocidas como apps de mensajería, aplicaciones o apps de chat (Camacho, Caldera y Valenzuela, 2019; Carrillo, Cascales y López, 2018; Henze, Pielot, Poppinga, Schinke y Boll, 2011). También están los podcast, archivos multimedia en audio o vídeo que incluyen texto ya sea como subtítulos o notas (Contreras, Herrera y Ramírez, 2009; Turró, Despujol y Busquets, 2014); por supuesto existen notables esfuerzos por incorporar las sumamente populares redes sociales a la educación a distancia (Belli y Reyes, 2015; Torres et al., 2012); los videojuegos son una vivencia significativa y viable en contextos de aprendizaje, ya que, en más de algún caso, es factible analizar su impacto en el desempeño académico, la motivación y el grado de satisfacción (Bossolasco, Enrico, Casanova y Enrico, 2015; Jiménez y Diez, 2018; Rodríguez, López y Mortera, 2017). Quedan por desarrollar en el entorno de la educación a distancia las notificaciones push, la inteligencia artificial y los asistentes de voz. 


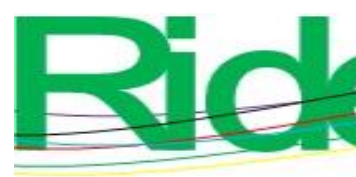

Revista Iberoamericana para la

Investigación y el Desarrollo Educativo

ISSN $2007-7467$

Tomando en consideración toda la información mencionada hasta ahora, se seleccionó como objeto de estudio al Instituto Politécnico Nacional (IPN), con la finalidad de responder la siguiente pregunta de investigación: ¿cómo se promueve el aprendizaje autónomo en alumnos de educación a distancia a través de recursos digitales? El objetivo es documentar experiencias del aprendizaje autónomo desde la perspectiva de los profesores de educación a distancia del IPN.

\section{Método}

La metodología que se utilizó fue cualitativa. La investigación fue no experimental con diseño transversal y exploratorio. Se seleccionó el estudio de caso como técnica para obtener la información, toda vez que es una investigación sistemática y empírica. La muestra fue no probabilística y heterogénea.

\section{Diseño de la muestra}

Se estructuró una muestra heterogénea compuesta de 12 profesores de centros de estudios científicos y tecnológicos y 8 de escuelas de educación superior del sistema de educación a distancia del IPN. Los participantes fueron seleccionados por su formación académica, su experiencia profesional y su labor docente en el IPN. Las entrevistas se realizaron entre los meses de marzo y diciembre del año 2018.

\section{Recolección de la información}

Una vez que se seleccionó a los posibles participantes de las entrevistas, se contactaron y se estableció fecha, hora y lugar de la entrevista; posteriormente se elaboró el programa de las entrevistas, y finalmente se procedió a realizarlas. Para hacer las entrevistas se diseñó un cuestionario con seis preguntas a profundidad, donde el entrevistador introdujo comentarios adicionales para precisar conceptos y obtener mayor información sobre el tópico estudiado. Cabe hacer mención que el cuestionario planteó una situación específica con la finalidad de que los profesores expusieran sus experiencias acerca de cómo promueven el aprendizaje autónomo en sus estudiantes de modalidad a distancia y el uso de recursos digitales. El planteamiento de la situación y las preguntas fueron las siguientes: 


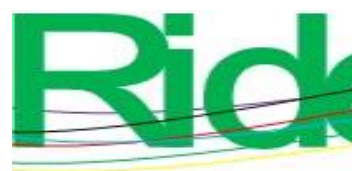

Revista Iberoamericana para la Investigación y el Desarrollo Educativo

ISSN $2007-7467$

La actividad cuatro de la unidad de aprendizaje que imparte señala retomar los resultados de la actividad tres como base para hacer un trabajo más elaborado, amplio y profundo. La finalidad es dar seguimiento a las habilidades y competencias logradas con anterioridad. A este respecto, uno de sus alumnos le comenta: "Me permito comunicarme con usted porque tengo dificultades para hacer la actividad cuatro, tengo el trabajo de la actividad tres, pero por causas de fuerza mayor ya no puedo dar seguimiento al mismo trabajo. ¿Puede darme la oportunidad de hacer la actividad cuatro con otra información? Estoy dispuesto a realizar todo el trabajo desde el principio".

Teniendo en cuenta esta eventualidad, responda las siguientes preguntas: ¿Qué respuesta le daría al alumno? ¿Permitiría cambios para realizar la actividad programada? ¿Cuáles serían las razones? ¿Cómo manejaría la situación con los otros alumnos? ¿Cuáles serían las ventajas de la nueva propuesta para conseguir las competencias en el alumno? ¿Se promueve el aprendizaje autónomo en los alumnos a través de recursos digitales?

\section{Procesamiento de la información}

El procesamiento de la información consistió en transcribir las entrevistas del archivo de audio en el procesador de texto Word; se generó un archivo por cada entrevista. Después se elaboró una síntesis de cada una para ser utilizada en la etapa de sistematización de la información.

\section{Sistematización y análisis de la información}

Con fundamento en Peinado, Cerecedo y Jaramillo (2015), la sistematización y análisis de la información se organizó de la siguiente forma:

Antes que nada, vale la pena señalar que a lo largo de esta etapa se utilizó la herramienta informática ATLAS.ti, cuya finalidad es facilitar el análisis cualitativo de datos textuales. Ahora bien, como primer paso se ingresaron los documentos primarios, a saber, las transcripciones de las entrevistas que se realizaron a los profesores. El segundo paso fue elaborar las citas de los documentos primarios. Las citas son segmentos significativos de estos documentos primarios, es la primera selección del material para la reducción de los datos de campo. El tercer paso fue codificar la información. Los códigos son conceptualizaciones, resúmenes o agrupaciones de las 


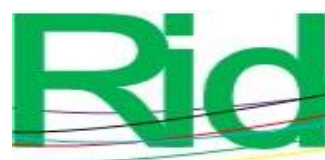

citas, lo que implica un segundo nivel de reducción de los datos. El cuarto paso fue procesar los memos o notas. Se trata de comentarios de un nivel cualitativamente superior, puesto que incluyen todas aquellas anotaciones que se realizaron durante el proceso de análisis: desde notas recordatorias hasta explicaciones de las relaciones encontradas, conclusiones y demás que pueden ser utilizadas como punto de partida para la redacción del informe. El quinto paso fue crear las megafamilias, superfamilias y familias, agrupaciones de códigos que tienen el fin de establecer las relaciones de diferente tipo entre los componentes (Peinado et al., 2015, p. 145).

La estructuración de megafamilias, superfamilias y familias se describen en la tabla 1. 
Tabla 1. Estructuración de megafamilias, superfamilias y familias

\begin{tabular}{|c|c|c|}
\hline Megafamilias & Superfamilias & Familias \\
\hline \multirow{8}{*}{$\begin{array}{l}\text { 1) La actividad } \\
\text { y su propósito }\end{array}$} & \multirow{2}{*}{$\begin{array}{l}\text { 1) Respuestas al } \\
\text { alumno }\end{array}$} & 1) Ratificar lo establecido en la actividad \\
\hline & & 2) Adecuar la actividad \\
\hline & \multirow{3}{*}{$\begin{array}{l}\text { 2) Argumentos para } \\
\text { no hacer cambios }\end{array}$} & 1) Cumplir con los objetivos en tiempo y forma \\
\hline & & 2) No afectar la actividad \\
\hline & & 3) Adquirir las competencias deseadas \\
\hline & \multirow{3}{*}{$\begin{array}{l}\text { 3) Justificaciones } \\
\text { para realizar } \\
\text { cambios }\end{array}$} & 1) Motivarlo a ser responsable y creativo \\
\hline & & 2) Considerar las circunstancias del alumno \\
\hline & & 3) Hacerlo por medios digitales \\
\hline \multirow{7}{*}{$\begin{array}{l}\text { 2) Manejo de la } \\
\text { situación }\end{array}$} & \multirow{2}{*}{$\begin{array}{l}\text { 1) Manejo del } \\
\text { problema }\end{array}$} & 1) Informar la situación del alumno al tutor \\
\hline & & 2) Apoyar la formación académica del alumno \\
\hline & \multirow{2}{*}{$\begin{array}{l}\text { 2) Postura con los } \\
\text { otros estudiantes }\end{array}$} & 1) Explicar razones para cambiar la actividad \\
\hline & & 2) Poner a discusión la situación del alumno \\
\hline & \multirow{3}{*}{$\begin{array}{l}\text { 3) Ventajas de una } \\
\text { nueva propuesta }\end{array}$} & 1) Lograr competencias en el alumno \\
\hline & & 2) Potenciar las capacidades del alumno \\
\hline & & 3) Fortalecer la dedicación del alumno \\
\hline \multirow{5}{*}{$\begin{array}{l}\text { 3) Aprendizaje } \\
\text { autónomo }\end{array}$} & \multirow{4}{*}{$\begin{array}{l}\text { 1) Aprendizaje } \\
\text { aplicado al entorno y } \\
\text { realidad del alumno }\end{array}$} & 1) Promover el pensamiento crítico y reflexivo \\
\hline & & 2) Permitir la autodirección y la autorregulación \\
\hline & & 3) Aumentar el aprendizaje significativo \\
\hline & & 4) Emplear habilidades metacognitivas \\
\hline & 2) Recursos digitales & $\begin{array}{l}\text { 1) Utilizar tecnologías de la información y la } \\
\text { comunicación (TIC) }\end{array}$ \\
\hline
\end{tabular}

Fuente: Elaboración propia

\section{Resultados}

A continuación se presentan los principales resultados obtenidos. En relación con la pregunta “¿Qué respuesta le daría al alumno?”, la mayoría de los profesores respondió que sí apoyarían al alumno con su solicitud. Algo que los encuestados mencionaron en más de una ocasión fue que, para dar solución a la problemática, el trabajo de acompañamiento por parte del tutor asignado es fundamental. En este sentido, algunos profesores coinciden en que el alumno debe 


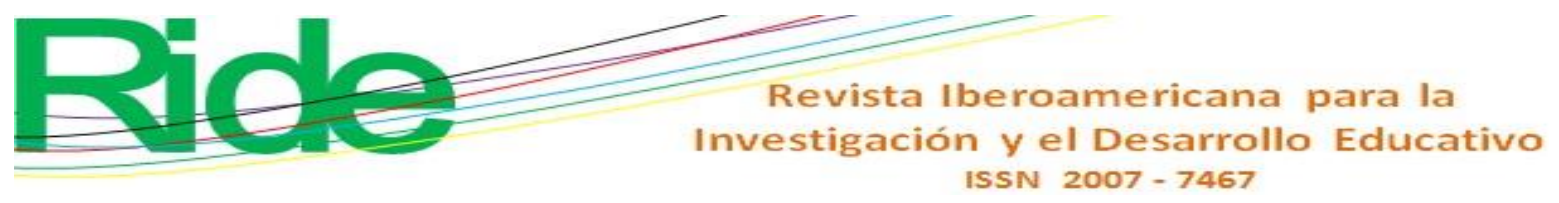

comunicarle al tutor cualquier cambio que realice, de domicilio o de trabajo, por ejemplo, para que este pueda tener la base de datos de sus tutorados actualizada. Asimismo, los profesores entrevistados coinciden en que el tutor debe conocer la trayectoria académica del alumno y obtener mayor retroalimentación a través de sus comentarios. Adicionalmente, expresaron que cambiar el lugar no es importante, que lo verdaderamente sustancial es desarrollar las habilidades para evaluar la competencia del estudiante.

Acerca de la pregunta “¿Permitiría cambios para realizar la actividad programada?”, la respuesta de la mayoría de los profesores es que sí le darían la oportunidad al estudiante de hacer la actividad en algún otro lugar. Entre las propuestas que se mencionan está la de ofrecer más tiempo para entregar el trabajo y así, con más tiempo, el alumno pueda efectuar la actividad. También enunciaron que se podría hacer con los resultados del trabajo anterior utilizando las TIC para lograrlo.

Referente a la pregunta “¿Cuáles serían las razones?”, los profesores exponen distintas razones. Una de ellas es que se respeta el propósito de la actividad. Los participantes concuerdan en que se debe apoyar a los alumnos cuando ellos mismos encuentran soluciones a sus problemáticas y de esta forma motivar su propio aprendizaje. Los entrevistados también indicaron que debe de existir flexibilidad tomando en consideración las circunstancias; reconocen que de esta manera también se promueve el aprendizaje autónomo y el desarrollo de las competencias señaladas en el programa.

Relativo a la pregunta “¿Cómo manejaría la situación con los otros alumnos?”, los docentes respondieron que explicarían las razones que existen para cambiar y adaptar la actividad del alumno en cuestión. Algunos de ellos argumentaron que lo consultarían mediante un foro o mensajería interna, para conocer si alguien más se encuentra en una situación semejante, hacer los ajustes necesarios en su grupo e informar al tutor, con el objetivo de ofrecer alternativas y que no se queden sin adquirir la competencia de la actividad. Únicamente dos profesores entrevistados indicaron que informarían a los alumnos si fuera realmente necesario, ya que consideraron que no se requiere un manejo especial de la situación con los demás integrantes del grupo.

Concerniente a la pregunta "¿Cuáles serían las ventajas de la nueva propuesta para conseguir las competencias en el alumno?", los profesores entrevistados establecieron que, además de promover el logro de las competencias de la actividad, se debe motivar el aprendizaje con herramientas tecnológicas y generar pensamiento crítico y reflexivo, así como emplear sus habilidades metacognitivas. También indicaron que la principal ventaja de la propuesta es que el 


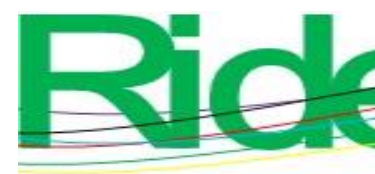

Revista Iberoamericana para la

Investigación y el Desarrollo Educativo

ISSN $2007-7467$

alumno desarrolle la autorregulación de su propio aprendizaje al cumplir con el objetivo de la actividad. Los entrevistados manifestaron que otra de las ventajas esperadas es fortalecer la autoestima, seguridad en sus decisiones, iniciativa y creatividad, las cuales impactarán en la motivación y dedicación del estudiante.

Correspondiente a la pregunta "¿Se promueve el aprendizaje autónomo en los alumnos a través de recursos digitales?", los participantes enlistaron diversas TIC para realizarlo. Se plantea el correo electrónico como medio para efectuar el trabajo solicitado: envían archivos y después reciben las respuestas por este mismo recurso. Proponen, asimismo, compartir por Skype, por vía telefónica tradicional, por WhatsApp o por redes sociales. Los profesores también mencionan Google Forms, medio por el cual es posible realizar encuestas, basta enviar un enlace a los estudiantes para que estos la puedan contestar, de esta manera se puede recopilar la información que se requiere. Por último, señalan Google Drive como recurso digital.

\section{Discusión}

Siguiendo a Gros (2016), “cuando las tecnologías y los diseños educativos se comunican, hay una interdependencia dinámica" (p. 2). Así pues, la clave para resolver la situación del alumno no es cambiar los contenidos de la unidad de aprendizaje, u ofrecer opciones distintas a cada estudiante; el objetivo es acceder a que se cumpla el propósito de la actividad de forma distinta para conseguir el mismo aprendizaje. Estas situaciones no deben ser observadas como un problema, sino como una extraordinaria oportunidad de promover el aprendizaje autónomo.

Romero y Barberà (2013) indican que "se debe permitir que el estudiante desarrolle sus competencias de gestión o regulación de los aprendizajes y que sepa conciliar sus diferentes tiempos profesionales, familiares y académicos" (p. 14). De esta forma, el aumento de la confianza y la autorregulación son resultados benéficos del proceso de aprendizaje a distancia, aplicando enfoques basados en la experiencia personal de los estudiantes (Gutiérrez y Gallego, 2017, p. 1181), y con ello los alumnos desarrollan el aprendizaje autónomo: aprender a aprender (MacDougall, 2008, p. 224). Al respecto, el alumno muestra habilidades de aprendizaje autónomo al proponer una alternativa a su problemática y responsabilizarse para cumplir con el trabajo. El profesor debe alentar y promover este tipo de aprendizaje, pero, al mismo tiempo, tiene que ser transparente con los otros estudiantes y con los lineamientos de la unidad de aprendizaje. Es pertinente recordar que el docente puede modificar las formas de trabajo, el tipo de trabajo 


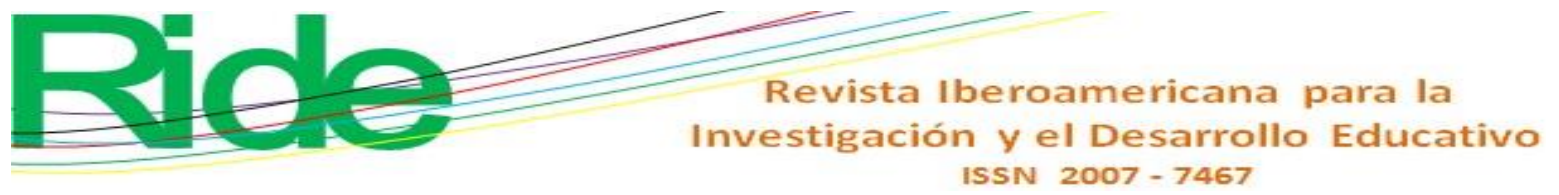

individual o en equipo, los tiempos de entrega, los instrumentos de evaluación y las fuentes de información. Lo que no es recomendable cambiar son las competencias que el alumno debe adquirir, los propósitos de la actividad y el porcentaje de evaluación.

Hanewicz et al. (2017) establecen que "la enseñanza centrada en el alumno es una pedagogía más efectiva para los estudiantes en línea” (p. 2). Los profesores que se enfocan en la promoción del aprendizaje autónomo en el estudiante orientan de forma adecuada los métodos pedagógicos, mejoran la comunicación, manejan apropiadamente los recursos disponibles y adaptan las actividades a las condiciones de vida de los estudiantes. Adicionalmente, cuando el profesor retroalimenta las actividades de sus alumnos, promueve el aprendizaje autónomo del estudiante, pues le permite reconocer sus fortalezas y áreas de mejora.

Se debe considerar la atención a los enfoques tecnológicos, las tendencias y los desarrollos actuales que permitan obtener información masiva y la difusión del conocimiento (Arancibia, Cabero y Valdivia, 2019, p. 104; Gros, 2016, p. 11; Molinero y Chávez, 2019, p. 9). Conviene decir que el profesor debe conocer el manejo y uso de las herramientas y medios digitales, sus habilidades técnicas deben comprender la abundancia, el análisis y la adaptabilidad. A través de ellos, debe lograr la individualización y la diferenciación para alcanzar la personalización de las actividades, con prácticas educativas inclusivas, democráticas y participativas, basadas en estrategias de autoplanificación y autorregulación, buscando la adquisición de competencias y habilidades para desarrollar el aprendizaje autónomo en los estudiantes.

Desde otro ángulo, Xiao (2012) determina que "la relación tutor-alumno es otro factor a considerar porque afecta la motivación del aprendizaje de los alumnos” (p. 375). En esa línea, la comunicación entre el profesor y tutor debe ser continua para acompañar la trayectoria académica del alumno.

En términos generales, la investigación evidenció que los profesores fomentan el aprendizaje autónomo al permitir que los alumnos intervengan en la construcción de procedimientos y recursos para alcanzar el propósito y las habilidades planteadas en una actividad específica, de esta manera el alumno toma conciencia sobre sus decisiones para dirigir y regular su propio aprendizaje.

El presente estudio generó datos para investigaciones más introspectivas, no se considera generalizable y tampoco es concluyente (Hernández, Fernández y Baptista, 2010, p. 165). Se recomienda para futuros trabajos ampliar la dimensión de la muestra, incluir experiencias de alumnos de educación a distancia, indagar sobre prácticas docentes y su transformación con apoyo 


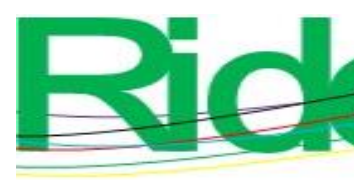

Revista Iberoamericana para la

Investigación y el Desarrollo Educativo

ISSN $2007-7467$

de las nuevas tecnologías, determinar la aceptación de la tecnologías de otros actores asociados al proceso de educativo, el papel que desempeñan las autoridades escolares y la trascendencia de la normatividad actual, así como el impacto financiero que representa el uso de estas tecnologías en el ámbito educativo.

\section{Conclusiones}

El objetivo del estudio fue contribuir con datos y experiencias sobre el aprendizaje autónomo desde el punto de vista de los profesores.

En el transcurso de sus estudios, los alumnos enfrentan distintas problemáticas y con frecuencia ellos mismos plantean propuestas para resolverlas. Esto habla de su nivel de creatividad y responsabilidad. En este aspecto, el profesor realiza cambios permitiendo que el alumno avance y adquiera las competencias necesarias para su formación. Pero antes de realizar cualquier cambio es recomendable examinar las implicaciones en el propósito de la actividad, la colaboración entre estudiantes y los recursos digitales a utilizar.

Los profesores entrevistados entienden que los alumnos utilizan medios digitales para enfrentar los retos que se les presentan en su aprendizaje, aún más cuando se trata de que ellos aprendan por sí mismos. Se debe permitir esta flexibilidad al momento de considerar la situación y circunstancias de cada alumno.

Promover el pensamiento crítico y reflexivo, permitir la autodirección y la autorregulación, aumentar el aprendizaje significativo y emplear habilidades metacognitivas son algunas de las ventajas que los profesores entrevistados perciben al momento de fomentar el aprendizaje autónomo en los alumnos.

Como línea de trabajo adicional, es posible considerar otros estudios de corte cuantitativo que se enfoquen en la eficiencia terminal, tal como se hace en algunos trabajos en la educación presencial (Peinado y Jaramillo, 2018; Peinado, Mayagoitia y Cruz, 2019), y determinar los factores que intervienen en la deserción de la modalidad a distancia. 


\section{Referencias}

Alqurashi, E. (2019). Predicting student satisfaction and perceived learning within online learning environments. Distance Education, 40(1), 133-148. Retrieved from http://doi.org/10.1080/01587919.2018.1553562.

Amador, C. y Velarde, L. (2019). Competencias para el uso de las TIC en estudiantes de educación superior: un estudio de caso. RIDE Revista Iberoamericana para la Investigación y el Desarrollo Educativo, 10(19), 1-27. Recuperado de https://doi.org/10.23913/ride.v10i19.515.

Arancibia, M., Cabero, J. y Valdivia, I. (2019). Estudio comparativo entre docentes y estudiantes sobre aceptación y uso de tecnologías con fines educativos en el contexto chileno. Apertura, 11(1), 104-119. Recuperado de http://dx.doi.org/10.32870/Ap.v11n1.1440.

Belli, S. y Reyes, M. (2015). La experiencia de lo virtual en la educación digitalizada. Cuestiones de confianza en contextos educativos online. RED-Revista de Educación a Distancia, 47(5), 1-21. Recuperado de http://www.um.es/ead/red/47/Belli.pdf.

Bossolasco, M., Enrico, R., Casanova, B. y Enrico, E. (2015). Kokori, un serious game. La perspectiva de los estudiantes ante una propuesta de aprendizaje innovadora. RED, Revista de Educación a Distancia, 45(3), 1-17. Recuperado de http://www.um.es/ead/red/45/bossolasco.pdf

Camacho, A., Caldera, M. y Valenzuela, V. (2019). Fidelidad en el uso de app para la resolución de ecuaciones diferenciales. Apertura, 11(1), 74-89. Recuperado de http://dx.doi.org/10.32870/Ap.v11n1.1463

Cardoso, E. y Cerecedo, M. (2019). Estudio evaluativo de las competencias genéricas en las licenciaturas de comercio y administración de modalidad virtual del IPN. RIDE. Revista Iberoamericana para la Investigación y el Desarrollo Educativo, 9(18), 62-83. Recuperado de https://doi.org/10.23913/ride.v9i18.411

Carrillo, M., Cascales, A. y López, A. (2018). Apps para el aprendizaje de idiomas en la Universidad de. RED. Revista de Educación a Distancia, 58(13), 1-18. Recuperado de http://dx.doi.org/10.6018/red/58/13

Chen, R., Bennett, S. and Maton, K. (2008). The adaptation of Chinese international students to online flexible learning: two case studies. Distance Education, 29(3), 307-323. Retrieved from http://doi.org/10.1080/01587910802395821 


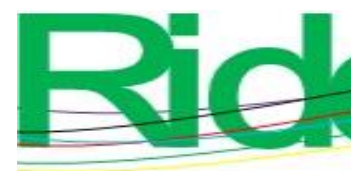

Revista Iberoamericana para la

Investigación y el Desarrollo Educativo

ISSN $2007-7467$

Cho, M.-H. and Shen, D. (2013). Self-regulation in online learning. Distance Education, 34(3), 290-301. Retrieved from http://doi.org/10.1080/01587919.2013.835770

Clarence, Ng. (2008). Multiple-goal learners and their differential patterns of learning. Educational Psychology, 28(4), 439-456. Retrieved from http://doi.org/10.1080/01443410701739470

Clarence, Ng. (2017). Distance learners' multiple goals, learning and achievement in different learning situations. Distance Education, 38(1), 37-58. Retrieved from http://doi.org/10.1080/01587919.2017.1298981

Contreras, J., Herrera, J. y Ramírez, M. (2009). Elementos instruccionales para el diseño y la producción de materiales educativos móviles. Apertura, 1(1), 84-99. Recuperado de http://www.udgvirtual.udg.mx/apertura/index.php/apertura/article/view/121/120

Domínguez, C., Organista, J. y López, M. (2018). Diseño instruccional para el desarrollo de contenidos educativos digitales para teléfonos inteligentes. Apertura, 10(2), 80-93. Recuperado de http://www.udgvirtual.udg.mx/apertura/index.php/apertura/article/view/1346/969

Esteban, M., y Zapata, M. (2016). Estrategias de aprendizaje y eLearning. Un apunte para la fundamentación del diseño educativo en los entornos virtuales de aprendizaje. RED. Revista de Educación a Distancia, 50(15), 1-12. Recuperado de http://www.um.es/ead/red/50/esteban_zapata.pdf

García, L. (2012). ¿Por qué va ganando la educación a distancia? (1a ed.) Madrid: UNED.

Gros, B. (2016). Retos y tendencias sobre el futuro de la investigación acerca del aprendizaje con tecnologías digitales. RED. Revista de Educación a Distancia, 50(10), 1-13. Recuperado de http://www.um.es/ead/red/50/gros.pdf

Grover, J. and Troja, A. (2014). Satisfaction and success in online learning as a function of the needs for affiliation, autonomy and mastery. Distance Education, 35(1), 90-105. Retrieved from http://doi.org/10.1080/01587919.2014.891427

Gutiérrez, E. y Gallego, M. (2017). Presencia social en un ambiente colaborativo virtual de aprendizaje: análisis de una comunidad orientada a la indagación. Revista mexicana de investigación educativa, 22(75), 1169-1186. Recuperado de http://www.comie.org.mx/documentos/rmie/v22/n075/pdf/75007.pdf

Hanewicz, Ch., Platt, A. and Arendt, A. (2017). Creating a learner-centered teaching environment using student choice in assignments. Distance Education, 38(3), 273-287. Retrieved from http://doi.org/10.1080/01587919.2017.1369349 


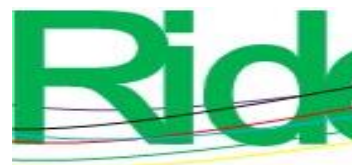

Revista Iberoamericana para la

Investigación y el Desarrollo Educativo

ISSN $2007-7467$

Henning, C. y Escofet, A. (2015). Construcción de conocimiento en educación virtual: Nuevos roles, nuevos cambios. RED, Revista de Educación a Distancia, 45(5), 1-10. Recuperado de http://www.um.es/ead/red/45/hennig.pdf

Henze, N., Pielot, M., Poppinga, B., Schinke, T. and Boll, S. (2011). My Apps is an experiment: Experience from User Studies in Mobile Apps Stores. International Journal of Mobile Human Computer Interaction, 3(4), 71-91. Retrieved from http://www.igiglobal.com/article/app-experiment-experience-user-studies/58926

Hernández, R., Fernández, C. y Baptista, P. (2010). Metodología de la investigación (1. ${ }^{a}$ ed.). México: McGraw-Hill.

Hromalik, C. and Koszalka, T. (2018). Self-regulation of the use of digital resources in an online language learning course improves learning outcomes. Distance Education, 39(4), 528-547. Retrieved from http://doi.org/10.1080/01587919.2018.1520044.

Jiménez, A. y Diez, E. (2018). Análisis del contenido de apps y videojuegos: implicaciones en procesos cognitivos en la lectura inicial. Apertura, 10(1), 71-87. Recuperado de http://dx.doi.org/10.32870/Ap.v10n1.1114.

MacDougall, M. (2008). Ten Tips for Promoting Autonomous Learning and Effective Engagement in the Teaching of Statistics to Undergraduate Medical Students Involved in Short-Term Research Projects. Journal of Applied Quantitative Methods, 3(3), 223-240. Retrieved from https://eric.ed.gov/?id=EJ813130.

Mendieta, A., Estrada, J. y Pérez, K. (2019). Millennials en torno a la comunicación y cultura para la educación superior. RIDE Revista Iberoamericana para la Investigación y el Desarrollo Educativo, 10(19), 1-24. Recuperado de https://doi.org/10.23913/ride.v10i19.497.

Molinero, M. y Chávez, U. (2019). Herramientas tecnológicas en el proceso de enseñanzaaprendizaje en estudiantes de educación superior. RIDE Revista Iberoamericana para la Investigación y el Desarrollo Educativo, 10(19), 1-31. Recuperado de https://doi.org/10.23913/ride.v10i19.494.

Niño, S., Castellanos, J. y Viloria, E. (2019). Construcción del conocimiento y regulación del aprendizaje en tareas colaborativas asíncronas. Apertura, 11(1), 6-23. Recuperado de http://dx.doi.org/10.32870/Ap.v11n1.1465.

Onrubia, J. (2016). Aprender y enseñar en entornos virtuales: Actividad conjunta, ayuda pedagógica y construcción del conocimiento. RED-Revista de Educación a Distancia, 50(3), 1-14. Recuperado de https://www.um.es/ead/red/50/onrubia.pdf. 


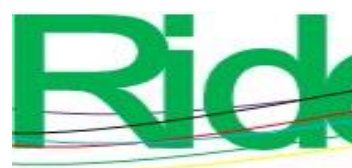

Revista Iberoamericana para la

Investigación y el Desarrollo Educativo

ISSN $2007-7467$

Park, S. and Yun, H. (2017). Relationships between motivational strategies and cognitive learning in distance education courses. Distance Education, 38(3), 302-320. Retrieved from https://doi.org/10.1080/01587919.2017.1369007.

Peinado, J., Cerecedo, M. y Jaramillo, D. (2015). Propuesta de un modelo de gestión del Capital Intelectual para los Centros de Investigación del IPN. Punto de Vista, 6(10), 135-157. Recuperado de http://dx.doi.org/10.15765/pdv.v6i10.768.

Peinado, J. y Jaramillo, D. (2018). La eficiencia terminal del Centro de Investigación e Innovación Tecnológica. Revista Electrónica de Investigación Educativa, 20(3), 126-134. Recuperado de https://doi.org/10.24320/redie.2018.20.3.1797

Peinado, J., Mayagoitia, V. y Cruz, C. (2019). Los grupos de investigación y su impacto en los factores que determinan la eficiencia terminal. Revista Dilemas Contemporáneos: Educación, Política y Valores, 7(1), 1-26. Recuperado de http://files.dilemascontemporaneoseducacionpoliticayvalores.com/200005708-

5b0b35b0b4/19.09.32\%20Los\%20grupos\%20de\%20investigaci\%C3\%B3n\%20y\%20su\% 20impacto\%20en\%201os\%20factores\%20que...pdf.

Rodríguez, R., López, B. y Mortera, F. (2017). El video como Recurso Educativo Abierto y la enseñanza de Matemáticas. Revista Electrónica de Investigación Educativa, 19(3), 92-100. Recuperado de https://doi.org/10.24320/redie.2017.19.3.936.

Romero, M. y Barberà, E. (2013). Identificación de las dificultades de regulación del tiempo de los estudiantes universitarios en formación a distancia. RED-Revista de Educación a Distancia, 38(1), 1-17. Recuperado de https://www.um.es/ead/red/38/romero_barbera.pdf.

Sun, S. (2016). Learntime and learning place-focused forward-oriented design for learning in technology-enhanced classrooms. Distance Education, 37(3), 349-365. Retrieved from http://doi.org/10.1080/01587919.2016.1233051.

Torres, J., Jara, D. y Valdiviezo, P. (2012). Integración de redes sociales y entornos virtuales de aprendizaje. RED-Revista de Educación a Distancia, 35(3), 1-8. Recuperado de https://www.um.es/ead/red/35/torres_et_al.pdf.

Turró, C., Despujol, I. y Busquets, J. (2014). Grabación automatizada de clases magistrales: el proyecto Videoapuntes de la UPV. RED-Revista de Educación a Distancia, 40(3), 1-7. Recuperado de https://www.um.es/ead/red/40/turro.pdf. 


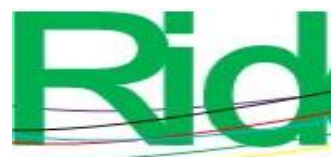

Revista Iberoamericana para la Investigación y el Desarrollo Educativo ISSN $2007-7467$

Xiao, J. (2012). Tutors' influence on distance language students' learning motivation: voices from learners and tutors. Distance Education, 33(3), 365-380. Retrieved from https://doi.org/10.1080/01587919.2012.723167. 